\title{
STED microscopy detects and quantifies liquid phase separation in lipid membranes using a new far-red emitting fluorescent phosphoglycerolipid analogue
}

\author{
Alf Honigmann, ${ }^{*}$ Veronika Mueller, Stefan W. Hell \\ and Christian Eggeling $\dagger^{*}$
}

Received 11th May 2012, Accepted 13th June 2012
DOI: 10.1039/c2fd20107k

We have developed a bright, photostable, and far-red emitting fluorescent phosphoglycerolipid analogue to probe diffusion characteristics of lipids in membranes. The lipid analogue consists of a saturated (C18) phosphoethanolamine and a hydrophilic far-red emitting fluorescent dye (KK114) that is tethered to the head group by a long polyethylenglycol linker. In contrast to reported far-red emitting fluorescent lipid analogues, this one partitions predominantly into liquid ordered domains of phase-separated ternary bilayers. We performed fluorescence correlation spectroscopy with a superresolution STED microscope (STED-FCS) to measure the lateral diffusion of the new lipid analogue in the liquid ordered (Lo) and disordered (Ld) phase. On a mica support, we observed micrometer large phases and found that the lipid analogue diffuses freely on all tested spatial scales $(40-250 \mathrm{~nm})$ in both the Ld and Lo phase with diffusion coefficients of $1.8 \mu \mathrm{m}^{2} \mathrm{~s}^{-1}$ and $0.7 \mu \mathrm{m}^{2} \mathrm{~s}^{-1}$, respectively. This indicates that the tight molecular packing of the Lo phase mainly slows down the diffusion rather than causing anomalous sub-diffusion. The same ternary mixture deposited on acid-cleaned glass forms Lo nanodomains of $<40 \mathrm{~nm}$ to $300 \mathrm{~nm}$ in diameter as only revealed by STED microscopy, which demonstrates the severe influence of interactions with the substrate on the sizes of domains in membranes. When averaging over different positions, STEDFCS measurements on such glass supported membranes displayed anomalous sub-diffusion. This anomaly can be attributed to a transient partitioning of the lipid analogue into the nano-domains, where diffusion is slowed down. Our results suggest that STED-FCS in combination with a Lo-partitioning fluorescent lipid analogue can directly probe the presence of Lo nano-domains, which in the future should allow the study of potential lipid rafts in live-cell membranes.

\section{Introduction}

Lipid phase separation into two liquid phases, namely the liquid ordered (Lo) and disordered (Ld) phase, has been extensively characterized in simple model membrane systems; for a review see for example, ref. 1 . The interest in the phase

Department of NanoBiophotonics, Max Planck Institute for Biophysical Chemistry, Göttingen, Germany.E-mail: ceggeli@gwdg.de (ChristianEggeling); ahonigm@gwdg.de (Alf Honigmann)

$\uparrow$ Current address: Human Immunology Unit, Weatherall Institute of Molecular Medicine, University of Oxford, U.K. 
behavior of lipid membranes can be mainly attributed to the lipid raftitheofiryicte Online proposing that liquid ordered domains made of sphingolipids, cholesterol and saturated phospholipids are critically involved in sorting and regulating proteins in the plasma membrane of eukaryotic cells. However, until now direct evidence for the existence of lipid rafts at physiological temperatures in the living cell is rare because of the lack of appropriate methods for detecting these small $(<200 \mathrm{~nm})$ and shortlived (ms) features. ${ }^{5-8}$ One widely-used approach is to study fluorescent lipid analogues in model and cell membranes with suitable microscopy techniques., ${ }^{9,10}$ Due to the putative small scale of lipid domains in cell membranes, established far-field fluorescence microscopy can give indirect information at best. With the development of super-resolution far-field microscopy, ${ }^{11,12}$ such as stimulated emission depletion (STED) microscopy ${ }^{13,14}$ the limitation in spatial resolution of far-field fluorescence microscopy given by the diffraction of light has been overcome and a spatial resolution down to $30 \mathrm{~nm}$ was attained in living cells. ${ }^{15}$ On the other hand, the high temporal resolution necessary for detecting diffusion kinetics of lipids in membranes on the sub-millisecond scale can be achieved by fluorescence correlation spectroscopy (FCS) using a photon counting detection scheme. ${ }^{16-18}$ By combining both STED and $\mathrm{FCS}^{19}$ it was for the first time possible to directly observe transient lipid interactions in live-cell membranes on the relevant spatial scales $<50 \mathrm{~nm} .^{15,20,21}$

To perform STED-FCS experiments, photostable and bright fluorescent lipid analogues should be used, featuring fluorescent dyes that emit in the far-red where absorption of biological material is minimal. However, the possible organic fluorophore labels are usually bulky and interfere with membrane packing when coupled to lipids. ${ }^{10}$ This may result in erroneous partitioning of the labeled lipid analogue, which was indeed observed for all potential Lo-partitioning lipid analogues labeled with a far-red emitting organic fluorophore. ${ }^{22}$ In fact, Lo-partitioning of the respective fluorescent lipid analogues was so low that STED-FCS could so far not be applied in the Lo phase on model membranes, leaving two important questions un-addressed: does the tighter molecular packing in the Lo phase only result in a slowed down yet normal diffusion, or is the diffusion 'anomalous' due to brief hindrances on the nanoscale, i.e. similar to the transient interactions observed for certain lipids in living cell membranes? ${ }^{15,20,21}$ Furthermore, can STED-FCS experiments detect transient incorporations of lipid analogues into nano-domains of higher molecular order such as putative lipid rafts in membranes of living cells?

Here, we address these questions by reporting the development of a far-red emitting fluorescent Lo-partitioning saturated phosphoglycerolipid analogue that is well suited for STED(-FCS) experiments. Using STED-FCS in ternary bilayers deposited on two different supports, specifically mica and glass, we studied the diffusion dynamics of the lipid analogue in both the Lo and Ld phase separately as well as its transient partitioning into nanometer sized domains. The results show that STED-FCS in conjunction with an appropriate fluorescent Lo-partitioning lipid analogue is an exceptional tool for detecting membrane nano-domains, including putative rafts in living cells.

\section{Results and discussion}

The measurement of nanoscopic lipid membrane organization and dynamics requires specific visualization of lipids. In the case of fluorescence microscopy this is realized by making the lipid luminescent. For example, most of the previous fluorescence microscopy measurements on lipid organization in model or cellular membranes have either applied a membrane-incorporating fluorescent dye, ${ }^{23}$ which however turns bright only in a liquid disordered environment and is not an analogue of a "real" lipid, or have labeled endogenous lipids by fluorescently tagged peptides or proteins, ${ }^{24,25}$ which however usually bind more than one lipid at a time (such as cholera-toxin B for the ganglioside lipid GM1) and may introduce biasing clustering. In our previous STED(-FCS) microscopy experiments, we have therefore realized 
fluorescent lipid analogues by adding a fluorescent label to the molieauktioffe Online interest. ${ }^{15,21}$ Unfortunately, it turned out that the bulky fluorophore labeled either at the lipid's head group or by acyl-chain replacement prevented the resulting lipid analogues from entering tightly packed areas of the membrane such as the Lo phase of phase separated model membranes. ${ }^{21,22}$ We concluded that a different labeling strategy where the label is placed further away from the membrane-water interface would avoid this limitation. Therefore, we synthesized a novel far-red emitting lipid analog DSPE-PEG-KK114 by tethering the organic fluorophore KK114 having an absorption and emission maximum of around 640 and $660 \mathrm{~nm},{ }^{26}$ respectively, to the head group of a saturated C18-phosphoethanolamine (1,2-dioctadecanoyl-sn- glycero-3-phosphoethanolamine, DSPE) by a long polyethylenglycol (PEG) linker (Fig. 1C). KK114 was preferred over the fluorophore Atto647N used in our previous studies, ${ }^{15,20,21}$ because it is more hydrophilic.

To characterize the partitioning, the diffusion and the photo-physical properties of the new lipid analogue, we used spin-coated lipid bilayers on a mica support. As a model system for phase separation we chose a three component mixture of 1,2-dipalmitoyl-sn-glycero-3-phosphocholine (DPPC), 1,2-diphytanoyl-sn-glycero3-phosphocholine (dPhyPC), and 30\% cholesterol which segregates into micrometer-large Ld and Lo domains at room temperature ${ }^{27,28}$ (Fig. 1A). dPhyPC was chosen because it is not exposed to (photo)oxidation as for example 1,2-dioleoylsn-glycero-3-phosphocholine (DOPC), and has also a low melting transition temperature. Bilayers were spin-coated on freshly cleaved mica from a solution with a lipid concentration of $2 \mathrm{~g} \mathrm{l}^{-1}$ in methanol-chloroform $(1: 1)$. As an Ld marker we used PE-Bodipy (PE-BDY). To show the influence of the PEG-linker on the partitioning of the DSPE analogue we also synthesized a lipid analogue with the dye KK114 directly attached to the head group (DSPE-KK114) ${ }^{26}$ (Fig. 1C).

The fluorescence images of the two different KK114-labeled DSPE lipid analogues showed that these analogues partitioned differently into the Ld and Lo phase (Fig. 1). DSPE-KK114 partitioned strongly in the same phase as PE-BDY, that is the Ld phase. In contrast, DSPE-PEG-KK114 partitioned predominantly in the Lo phase. A closer analysis of the fluorescence emission in the respective phases yielded a Lopartitioning coefficient $p_{L o}=5 \%$ for DSPE-KK 114 and $p_{L o}=70 \%$ for DSPE-PEGKK114, i.e. $70 \%$ of the fluorescent molecules partitioned into the Lo phase, rendering a Lo : Ld image contrast of $2.5: 1$. The fluorescence lifetime of the new Lo marker was 3.5-3.6 ns in both the Ld and Lo phase (Fig. 2A). A fluorescence lifetime that is independent on the phase partitioning of the lipid analogue shows that the fluorophore is not influenced by the different packing and hydration of the membrane in the Ld and Lo phase. Further, the lifetime of 3.5-3.6 ns is in the range of that of KK114 in aqueous solution ${ }^{26}$ and shorter than the 4 ns observed for the hydrophobic environment of the dye label when directly tagged at the head-group or by acyl-chain replacement. ${ }^{29}$ We attribute this finding to the fact that the fluorophore is kept out of the hydrophobic bilayer by the PEG-linker. Additionally, confocal FCS experiments (Fig. 2B) confirmed partitioning-independent fluorescence emission, because the molecular brightness of the fluorophore was similar in both phases $(\sim 18 \mathrm{kHz}$ photon counts with $7 \mu \mathrm{W}$ of $640 \mathrm{~nm}$ wavelength excitation light). The lateral diffusion coefficient determined in the Ld and Lo phase by confocal FCS was $D_{L d}=1.8 \mu \mathrm{m}^{2} \mathrm{~s}^{-1}$ and $D_{L o}=0.7 \mu \mathrm{m}^{2} \mathrm{~s}^{-1}$, respectively. The diffusion coefficient of DSPE-KK114 is similar in the Ld phase $\left(D_{L d}=1.9 \mu \mathrm{m}^{2} \mathrm{~s}^{-1}\right)$ and not determinable in the Lo phase due to the low partitioning into this phase. Note that the difference between $D_{L d}$ and $D_{L o}$ is $20 \%$ smaller than what has been reported in a previous study on fluorescent lipid analogues for separated Lo-Ld phases on mica. ${ }^{30}$ However, our experiments are not directly comparable to these previous studies because the ternary mixtures are different. The properties of the new lipid analogue are summarized in Fig. 2C.

To address the important question whether the diffusion of the Lo marker in large scale phase separated membranes is free or anomalous we measured FCS data of DSPE-PEG-KK114 diffusion in each phase for different observation areas with 
A
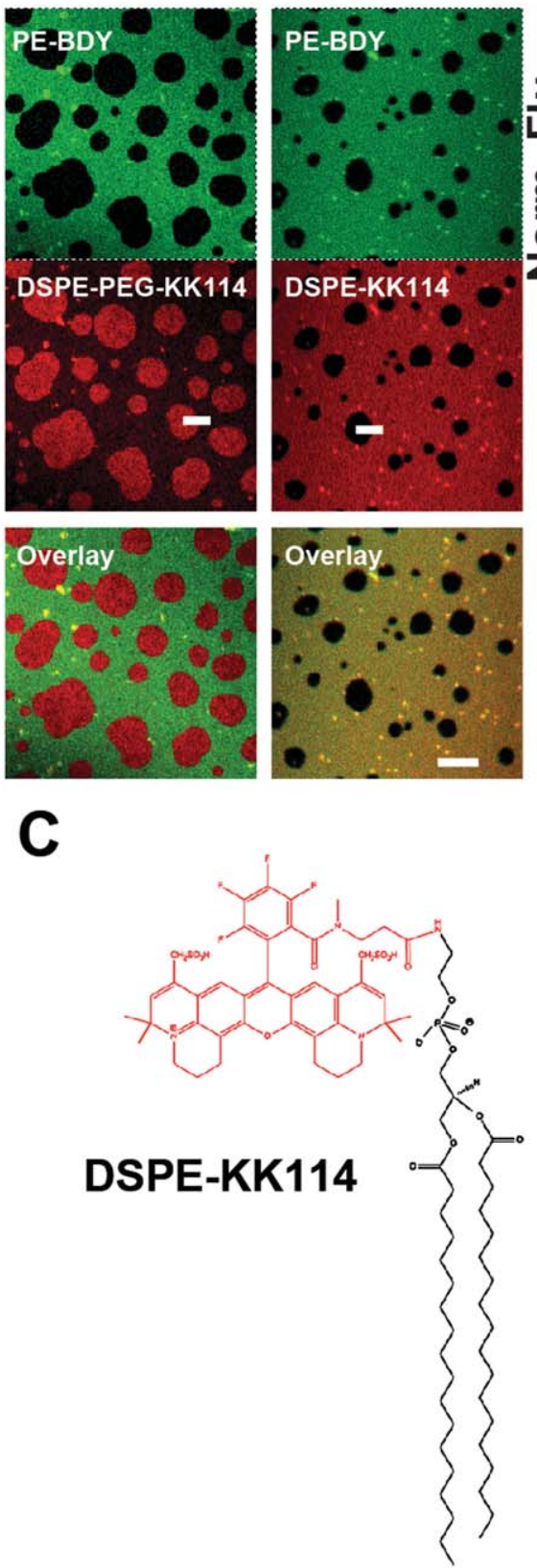

B

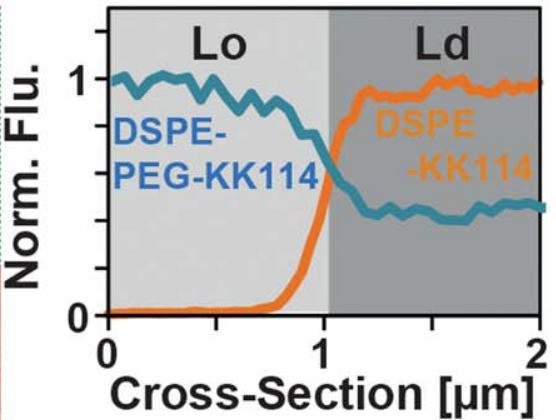

DSPE-PEGKK114

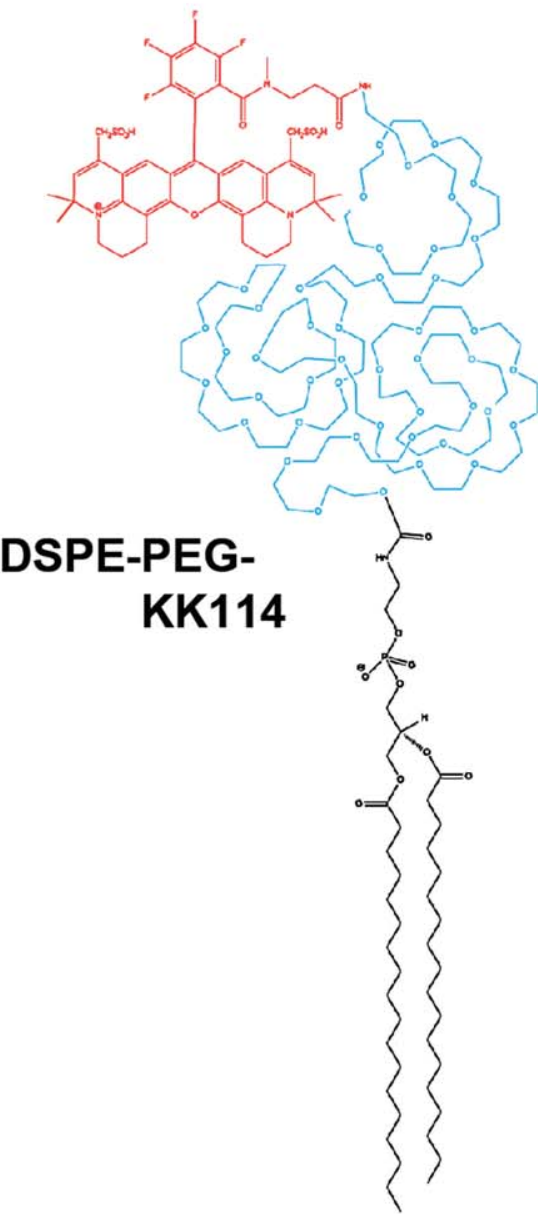

Fig. 1 Partitioning of DSPE-KK114 and DSPE-PEG-KK114 in ternary lipid bilayers on mica support. (A) Confocal two-color images showing the Ld marker PE-BDY in green (upper panels), and DSPE-KK114 (middle right) and DSPE-PEG-KK114 (middle left) in red. The overlays of the Bodipy and KK114 images (lower panels) show that DSPE-KK114 is excluded from the Lo phase whereas DSPE-PEG-KK114 is enriched. Scale bar: $2 \mu \mathrm{m}$. (B) Representative fluorescence intensity cross-section profiles (orange: DSPE-KK114, blue: DSPE-PEG-KK114) through domain boundaries (indicated by the lines in the middle images of A) highlighting the opposite partitioning pattern of the probes. (C) Structure of DSPE-KK114 and DSPE-PEGKK114. 
diameters $d$ ranging from diffraction-limited $250 \mathrm{~nm}$ down to sub-diffractionwalueke Online $d=40 \mathrm{~nm}$. The diameters $d$ were adjusted by varying the intensity of the STED beam in the focal region ${ }^{15,20,21}$ (compare Fig. 3A, B). These STED-FCS measurements directly revealed the dependence of the average molecular transit time $\tau_{D}$ through the observation area as a function of $d^{2}$ (extracted from FCS measurements the dependence $\tau_{D}\left(d^{2}\right)$ has previously been named FCS diffusion "law"18 or spotvariation $\mathrm{FCS}^{31}$ ). In case of free diffusion $\tau_{D}$ scales linearly with $d^{2}$, intersecting at the zero origin point, while hindered diffusion due to transient slowdowns (for example from trapping events or transient nano-domain incorporation) leads to a nonlinear dependence on $d^{2}$ with a positive intersect. ${ }^{18}$ Another way to illustrate this relationship is to calculate the average apparent diffusion coefficient $D=d^{2} /\left(8 \ln (2) \tau_{D}\right)$ and plot the values $D$ against the observation diameter $d .^{21}$ Constant values $D(d)$ on all spatial scales indicate free diffusion. Any significant deviations from constant values indicate complex diffusion as discussed in more detail later. Note, that $D$ is an apparent diffusion coefficient that may be an average over a multitude of diffusion modes and may not reveal sufficient insights into the diffusion characteristics when determined only for one observation diameter $d$; such insights are only revealed when assembling the dependence $D(d)$.

The function $D(d)$ measured by STED-FCS of DSPE-PEG-KK114 diffusion for the micrometer large phases on the mica support are depicted in Fig. 3C. In both the Ld and Lo phases the values $D(d)$ remained constant with decreasing $d$, which demonstrates free Brownian motion for both phases within the observed scales. Therefore, the approx. 2.6 times slower diffusion in the Lo phase stems from the increased molecular packing and increased viscosity, but not from hindrances on the tens of nanometer scale. Thus, at least for the mica support and on the observed scales $(>40 \mathrm{~nm})$, the segregation of the lipids is relatively unhindered resulting in homogenous Lo and Ld phases.

We in addition determined the anomaly coefficient $\alpha$ from the STED-FCS data. Values of $\alpha \approx 1$ reveal a homogeneous diffusion of all molecules, while $\alpha<1$ indicates heterogeneous sub-diffusion, i.e. strong differences in transit times between different molecules. ${ }^{17,32}$ Using STED-FCS, we have for example previously determined the dependence of the anomaly coefficient $\alpha$ on the diameter $d$ of the observation area for different fluorescent lipids in the plasma membrane of living cells. ${ }^{15}$ For some of the lipids the decrease of $\alpha$ towards small $d$ revealed transient trapping on nanoscales. At large $d$, FCS data revealed $\alpha \approx 1$, meaning that the anomalous sub-diffusion due to transient trapping events was averaged out at the large detection spots and therefore diffusion seemed misleadingly free. Only for small observation areas we could distinguish between dissimilar modalities of transits: a modality where the lipid happens to diffuse freely and another modality with a more complex transit time stemming from hindered diffusion due to a trapping event. In our present experiments, we determined $\alpha \approx 1$ for DSPE-PEG-KK114 in Ld and Lo on all spatial scales $d=40-250 \mathrm{~nm}$ (Fig. 3D), confirming our above observations of a homogeneous diffusion in both phases.

We note that the diffusion of DSPE-PEG-KK114 in the Ld phase of our ternary mixture was significantly slower $\left(1.7 \mu \mathrm{m}^{2} \mathrm{~s}^{-1}\right)$ than in the complete liquid disordered environment of a one component DOPC bilayer $\left(9 \mu \mathrm{m}^{2} \mathrm{~s}^{-1}\right)$ and even slower than in a DOPC bilayer containing 30\% cholesterol $\left(2.6 \mu \mathrm{m}^{2} \mathrm{~s}^{-1}\right)$ (grey lines in Fig. 3C). Besides slight differences in mobility from using dPhyPC instead of DOPC, this may indicate that the Ld phase of our ternary membranes still contained cholesterol and saturated lipids (DPPC), which both slow down diffusion by increasing the order and thus viscosity. The fact that the saturated lipid may also partition to the Ld phase suggests that the still incomplete partitioning of our Lo lipid analogue $\left(p_{L o}=70 \%\right)$ may actually resemble the partitioning characteristics of a non-labeled saturated lipid with a minimal influence by the dye label.

So far, large scale phase separation has not been observed in the plasma membrane of intact living cells. Yet, the lipid-protein ensemble of the plasma 

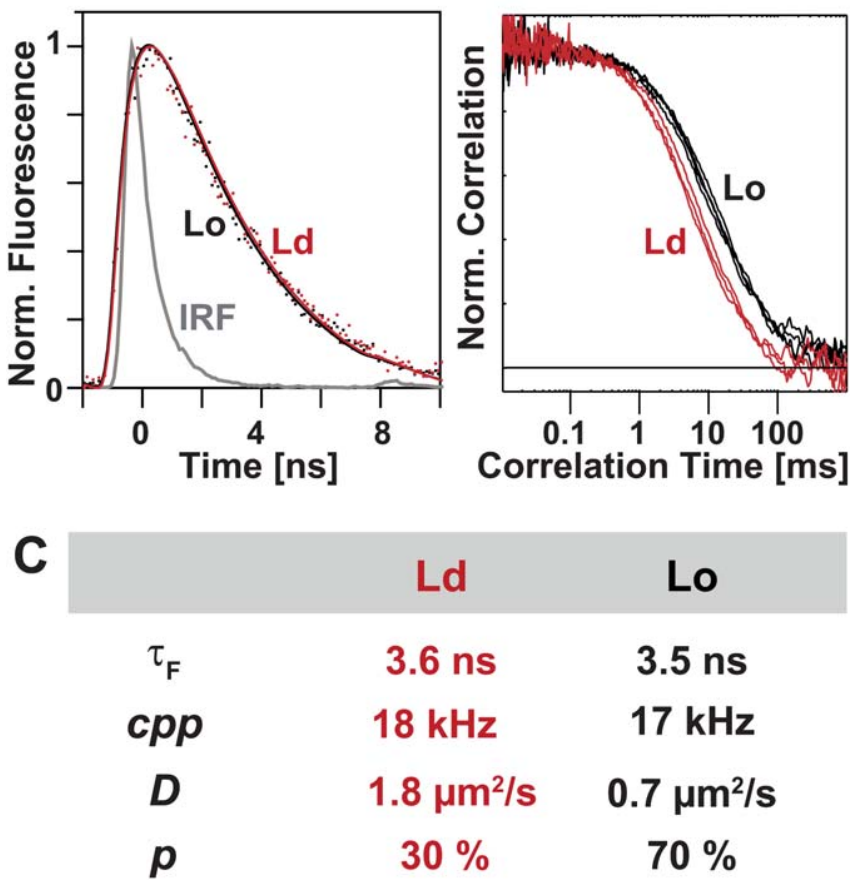

Fig. 2 Properties of DSPE-PEG-KK114 in the lipid bilayers. (A) The fluorescence lifetime does not depend on the lipid environment: representative TCSPC fluorescence lifetime histograms in the Lo (black) and Ld (red) phase (dots: experimental data, lines: fit, grey: instrument response function IRF). (B) Diffusion of DSPE-PEG-KK114 is slowed down in the Lo phase: representative confocal FCS data $\mathrm{G}\left(t_{c}\right)$ in the Lo (black) and Ld (red) phase. (C) Summarizing table: fluorescence lifetime $\tau_{F}$, molecular brightness or counts-per-particle $c p p$ at $7 \mu \mathrm{W}$ excitation power, lateral diffusion coefficient $D$, and partitioning coefficient $p$ of DSPE-PEG-KK114 in the Lo and Ld phase.

membrane is able to phase separate into large domains when the membrane is detached from the cytoskeleton by chemical treatment. ${ }^{33,34}$ In this respect, large scale phase separation (as observed with ternary mixtures on mica or in giant unilamellar vesicles) may not be a suitable model system for potential nano-sized rafts in intact living cells. However, it is known that ternary mixtures do not segregate in large phases when glass is used as the support instead of mica. The reason is an asymmetry of the bilayer leaflets due to an interaction of the lower leaflet lipids with the rough glass surface. Simulations of domain formation on solid supports showed that in the lower leaflet domain, growth is prevented by diffusion restriction due to high friction and interaction with the substrate. The upper leaflet is coupled to the lower leaflet which restricts domain sizes and also pins domain positions. ${ }^{35}$ We used this simple system as the model for a lipid membrane with complex heterogeneities on the nanometer scale. Confocal images of spin-coated ternary bilayers of the same kind as before (DPPC, dPhyPC and cholesterol) on acid cleaned glass showed that the Ld (PE-BDY) and Lo marker (DSPE-PEG-KK114) were patterned in a counterstained fashion, which still indicates Lo and Ld phase separation (Fig. 4A). As expected, the domain sizes were much smaller than those on the mica support and below the diffraction limit, i.e. not fully resolvable by the diffraction-limited confocal recordings. We therefore used the STED microscope with a spatial resolution tuned to about $40 \mathrm{~nm}$ to record more detailed images of the distribution of the DSPEPEG-KK114 lipid analogue. The STED images showed irregularly shaped domains 


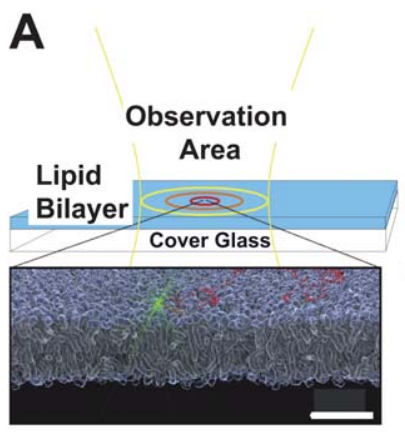

B
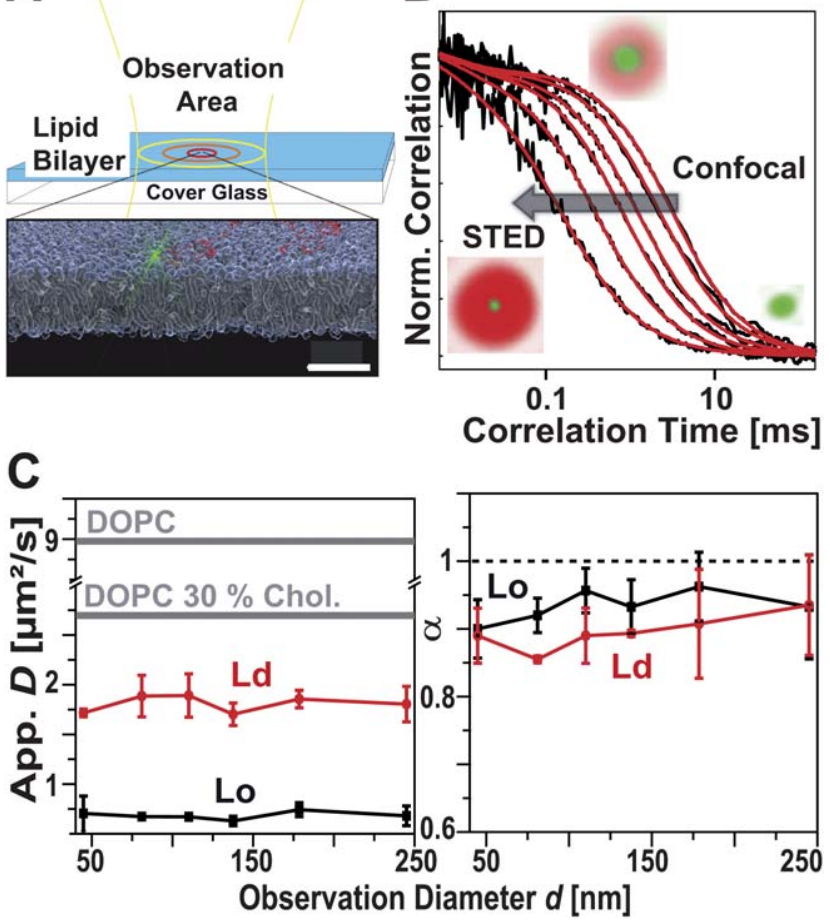

Fig. 3 Characterization of lipid dynamics in ternary bilayers on mica support. (A) Principle of STED-FCS on supported membranes: The diameter $d$ of the observation area is decreased by increasing the intensity of the doughnut-shaped STED beam. This allows the determination of the apparent diffusion coefficient $D(d)$ on spatial scales from $d=250 \mathrm{~nm}$ down to $40 \mathrm{~nm}$. (B) Representative STED-FCS data $G\left(t_{c}\right)$ of DSPE-PEG-KK114 recorded on the one-component DOPC bilayer for increasing STED power, exemplifying the decrease of the transit times with decreasing observation area. Insets: Schemes of the focal distribution of the region of emission (green) and inhibition (red) of fluorescence with increasing STED beam power. (C) Dependency of the apparent diffusion coefficient $D$ on $d$ of DSPE-PEG-KK114 on mica (black: Lo phase, red: Ld phase). For comparison, the grey lines indicate the diffusion coefficients of DSPE-PEGKK114 in a pure DOPC bilayer on mica without and with $30 \%$ cholesterol. (D) Anomaly coefficient $\alpha$ of DSPE-PEG-KK114 diffusion as a function of $d$ for Ld (red) and Lo (black).

with diameters from below $40 \mathrm{~nm}$ up to $300 \mathrm{~nm}$ and an average diameter of $90 \mathrm{~nm}$ (Fig. 4A). The latter value may however be smaller since we could not properly include dark domains which probably were of $<40 \mathrm{~nm}$ in diameter. As judged from time lapse imaging, the resolvable domains did not change their position or size over a period of $2 \mathrm{~h}$. Note that STED images of the DSPE-PEG-KK114 lipid in a single-component DOPC bilayer did not reveal any signs of domain formation (lower panel Fig. 4A).

We next applied STED-FCS to probe the diffusion of the DSPE-PEG-KK114 lipid in these heterogeneous membranes on scales between $250 \mathrm{~nm}$ to $40 \mathrm{~nm}$. From theory, we expect fast diffusion in the Ld areas in-between the nano-domains (similar to $D_{L d}=1.8 \mu \mathrm{m}^{2} \mathrm{~s}^{-1}$ on mica) and slow diffusion once the lipid analogue incorporates into the nano-domains (similar to $D_{L o}=0.7 \mu \mathrm{m}^{2} \mathrm{~s}^{-1}$ on mica). Due to several reasons, we could not determine the $D(d)$ dependence for the $\mathrm{Ld}$ and Lo portions separately: (1) the density of the nano-domains was too high and the sizes of most of them too small to accomplish all necessary STED-FCS measurements $(d=40-250 \mathrm{~nm})$ on a pure phase. (2) An exact positioning of the observation area on specific nano-domains was not feasible, since the FCS experiments required 
a relatively low concentration $(0.01 \mathrm{~mol} \%)$ of fluorescent lipid, whichiewastr(iine Online contrast to the micro-meter sized domains on mica) not high enough to realize an image contrast to accurately identify the nano-domains. We therefore recorded the $D(d)$ dependence at various random positions on the membrane, i.e. we probed the diffusion characteristics for different arrangements of domains with respect to the observation area. Fig. 4B shows the dependence $D(d)$ for three different random positions in the membrane. Clearly, the observed apparent diffusion coefficients were dependent on the position in the sample and ranged from $D=0.5 \mu^{2} \mathrm{~s}^{-1}$ to $2.5 \mu \mathrm{m}^{2} \mathrm{~s}^{-1}$. This variation and the fact that all of the observed $D(d)$ dependencies showed a deviation from a constant value indicated that the diffusion of the DSPE-PEG-KK114 lipid was heterogeneous in the phase-separated ternary bilayer on glass. This is supported by the decrease of the anomaly coefficient $\alpha$ with $d$ as plotted in Fig. 4C, indicating anomalous molecular diffusion on the nanoscale.

This anomaly in diffusion is to be expected, since we randomly positioned our observation area on the membrane, i.e. the probability to simultaneously probe diffusion inside and outside a domain is rather high. Consequently, on its diffusion path through the observation area the lipid may change from fast to slow diffusion and/or reverse, i.e. it is kind of trapped in the nano-domains. On one hand, this indicates that (as mentioned before) the values of $D$ only resemble an average over different diffusion modes and thus do not reveal a real diffusion coefficient. On the other hand, this diffusion characteristic is similar to the previously mentioned hindered diffusion of certain lipids in the plasma membrane of living cells, where STED-FCS revealed transient $(\approx 10 \mathrm{~ms}$ long) trapping. These traps indicated complexes with other relatively slow-moving or immobilized molecules such as proteins. ${ }^{15,20,21}$ Most importantly, the molecules hardly moved during the trap. ${ }^{15,36}$ However, this characteristic is different to the diffusion characteristics expected for the incorporation into nano-domains, during which the molecule still can move. Indeed, the deviations from homogeneous diffusion observed for the DSPE-PEG-KK114 lipid in the ternary mixtures on glass support were different from those observed for certain lipids in the plasma membrane of living cells. In the latter STED-FCS experiments, the transient trapping on molecular scales led to a continuous decrease of the values of $D$ (4.5 to 5-fold) when moving from $d=$ $250 \mathrm{~nm}$ down to $40 \mathrm{~nm} \cdot{ }^{15,20,21}$ In our current measurements, the decrease of $D$ was neither as strong (only up to 1.5 ) nor continuous. We rather observed an initial 1.5 -fold decrease for STED-FCS measurements between $d=250 \mathrm{~nm}$ and $\approx 150$ $\mathrm{nm}$, while values of $D$ stayed constant for smaller observation diameters $d$ and sometimes even increased again for very small $d$ (Fig. 4B). We can indicate that this is the $D(d)$ dependence expected for transient incorporation into nano-domains where diffusion is slowed down: (1) for diameters $d$ larger than the domain size, STEDFCS probes similar diffusion pathways for all molecular transits, which include both slow and fast diffusion components from inside and outside the Lo domains, respectively. Hence, the values of the apparent diffusion coefficient are smaller than the value $D_{\mathrm{Ld}}=1.8 \mu \mathrm{m}^{2} \mathrm{~s}^{-1}$ expected for the $\mathrm{Ld}$ phase (compare results from the mica support) and $\alpha \approx 1$. (2) With $d$ reaching the size of the nano-domains the molecular pathways become increasingly different with some of the molecules experiencing domain incorporation, i.e. slow-down of diffusion, and some not, which obviously leads to a slight decrease of the apparent $D$ and to $\alpha<1$. (3) For $d$ smaller than the domain sizes, the values of the apparent diffusion coefficient $D$ will either report the diffusion in- or outside of the domains dependent on the position of the observation area relative to the domain. In theory, one should in this case be able to extract two diffusion components (fast Ld and slow Lo diffusion) from these STED-FCS data. In practice, this was not feasible since the domain sizes were highly heterogeneous including the mentioned very small $<40 \mathrm{~nm}$ large nanodomains, all in all fading a clear separation of purely slow and purely fast molecular transits. (4) The experimentally observed dependence $D(d)$ is similar to that determined from Monte Carlo simulations of transient partitioning into nanometer-sized 
A

PE-BDY

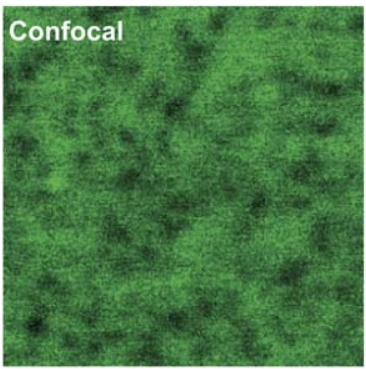

Confocal

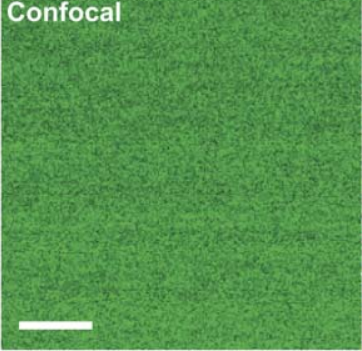

B

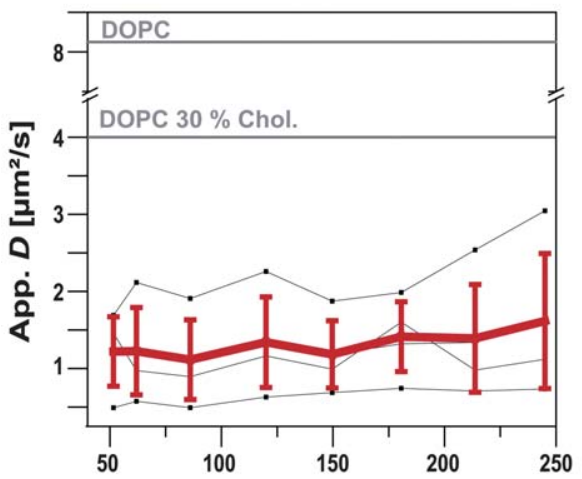

DSPE-PEG-KK114
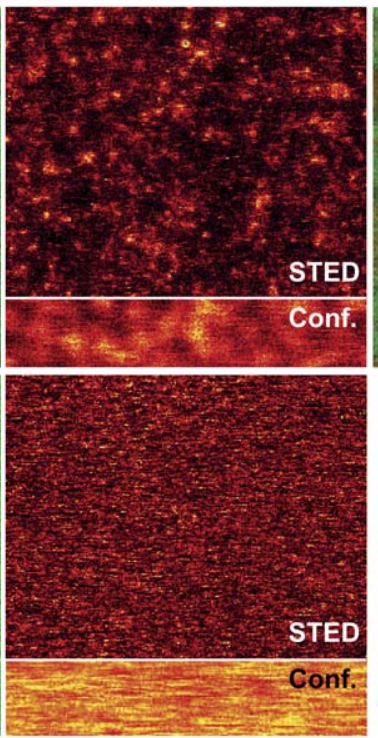

C
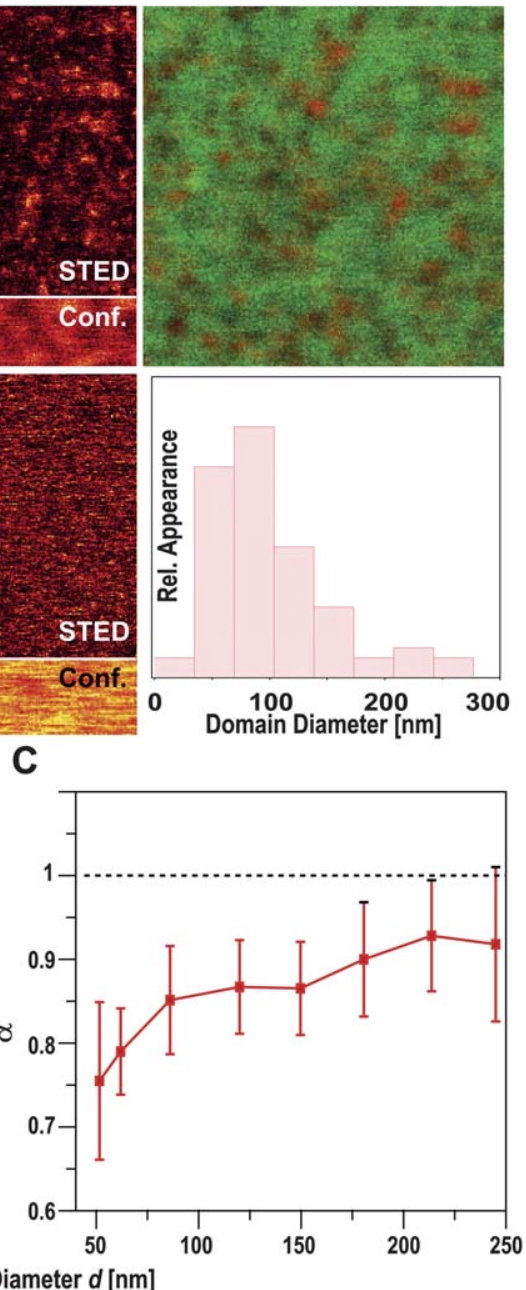

Fig. 4 Nano-domain partitioning in ternary bilayers on glass. (A) Scanning fluorescence images of the Ld-marker PE-BDY (green, left, confocal) and the Lo-marker DSPE-PEGKK114 (red, middle, upper parts STED and lower parts confocal recordings) in the ternary bilayer (upper images, showing domains) and a one-component DOPC bilayer (lower images, no domains). Scale bar: $1 \mu \mathrm{m}$. Upper right panel: Overlay of the BDY and KK114 images for the ternary mixture. Lower right panel: Frequency histogram of the diameters of the domains estimated from the DSPE-PEG-KK114 STED image on the ternary mixture with an average of $90 \mathrm{~nm}$ (total number of evaluated domains was 100). (B,C) STED-FCS analysis of DSPE-PEGKK114: Dependence of the apparent diffusion coefficient $D(B)$ and of the anomaly coefficient $\alpha(\mathrm{C})$ on the diameter $d$ of the observation area. Black lines depict representative measurements on three different random positions (for two membrane preparations) and the red lines the average over 10 measurements. For comparison, the grey lines indicate the diffusion coefficients of DSPE-PEG-KK114 in a pure DOPC bilayer on glass without and with $30 \%$ cholesterol.

domains in which diffusion is slowed down ${ }^{15,18}$ (however, in that case a constant domain size had been assumed).

The average $D(d)$ dependence obtained from averaging 10 independent STEDFCS measurements on different random positions also exhibits a slight decline of the apparent $D$ as $d$ was decreased (Fig. 4B red), however with a lower slope 
compared to some of the single measurements (Fig. 4B black). Averaging Aovere Online multiple positions seems to conceal the existence of the heterogeneous diffusion due to nano-domain partitioning. This averaged scenario is expected for the transient incorporation of molecules into putative nano-sized rafts in live-cell membranes, since these nano-domains are thought to be highly dynamic.

\section{Conclusion}

We have designed a novel far-red emitting fluorescent phosphoglycerolipid analogue DSPE-PEG-KK114 with suitable photophysical properties and causing minimal perturbations of the saturated lipid part. Most importantly, this fluorescent lipid analogue partitions into the Lo phase of membrane bilayers like its natural counterpart. This makes DSPE-PEG-KK114 a suitable candidate for studying phase separation and lipid diffusion characteristics in model membranes using STED(-FCS) microscopy. Here, we used DSPE-PEG-KK114 in conjunction with STED(-FCS) microscopy to study ternary mixtures in model membranes on mica as well as glass support. While phase separation into micrometer-sized Ld and Lo domains on the mica support could be observed with standard diffraction-limited microscopy, the superior spatial resolution of STED microscopy was necessary to resolve the $<40$ $\mathrm{nm}$ to $300 \mathrm{~nm}$ large Lo domains on the glass support. As expected, interactions of lipids of the lower leaflet of the membrane bilayer with the rough glass surface prevented the formation of large-scale domains. These nano-domains were confirmed when using STED-FCS to determine the diffusion characteristics of the lipid.

Most importantly, we could for the first time show that on scales ranging from $250 \mathrm{~nm}$ down to $40 \mathrm{~nm}$, diffusion is free in both the Ld and Lo phases. The about 2.6 times slower diffusion in the Lo phase results from the increased molecular packing and thus increased viscosity, but not from random hindrances at the tens of nanometer scale. Thus, we found that (at least on $\geq 40 \mathrm{~nm}$ spatial scales) segregation of the lipids is relatively unhindered resulting in homogenous Lo and Ld phases. This was directly evident for the micrometer large domains on the mica support. Using the Lo-partitioning lipid analogue on the glass support, we for the first time on the relevant spatial scales could report on the diffusion characteristics obtained for STED-FCS for transient nano-domain incorporation.

In conclusion, we have shown that STED-FCS in conjunction with the novel Lopartitioning fluorescent phosphoglycerolipid analogue is able to characterize transient incorporations into nano-domains of higher molecular order. This major development step should allow the study of more complex and dynamic systems such as critical fluctuations in ternary model membranes ${ }^{37}$ and, most importantly, potential nano-domain organizations and dynamics in live-cell membranes. ${ }^{2-4}$

\section{Material and methods}

\section{Lipids}

1,2-Dioleoyl-sn-glycero-3-phosphocholine (DOPC), 1,2-dipalmitoyl-sn-glycero-3phosphocholine (DPPC), 1,2-diphytanoyl-sn-glycero-3-phosphocholine (diPhyPC), cholesterol (CO), 1,2-dioctadecanoyl-sn-glycero-3-phosphoethanolamine (DSPE), 1,2-distearoyl-sn-glycero-3-phosphoethanolamine- $N$-[amino(polyethylene glycol)2000] (DSPE-PEG-Amine) were purchase from Avanti (Avanti Polar Lipids, Inc., Alabaster, Alabama USA).

\section{Fluorescent lipid analogue synthesis}

DSPE-KK114 was prepared from $1 \mathrm{mg}$ of the NHS ester of the fluorescent dye KK114 $(1 \mu \mathrm{mol})^{26}$ and $1.5 \mathrm{mg}$ of 1,2-stearoyl-sn-glycero-3-phosphoethanolamine $(2 \mu \mathrm{mol})$ in a solvent mixture consisting of chloroform, $N, N$-dimethylformamide and methanol $(2: 1: 1)$. Starting materials were dissolved in $0.5 \mathrm{~mL}$ of this solvent 
mixture, $10 \mu \mathrm{L}(70 \mu \mathrm{mol})$ of triethylamine was added, and the solution wasustiriede Online under argon at room temperature overnight. Then the solvents were evaporated in vacuo, and the product was isolated on a HPTLC plate with silica gel $(10 \times 10 \mathrm{~cm}$, $0.2 \mathrm{~mm}$ layer; VWR International; Darmstadt, Germany) using a chloroform-methanol-water mixture (65/35/8) as an eluent. MS (ESI): $\mathrm{m} / \mathrm{z}$ (negative mode, $\%$ ) = $807.4(100 \%)[\mathrm{M}-2 \mathrm{H}] 2-(\mathrm{C} 83 \mathrm{H} 121 \mathrm{~F} 4 \mathrm{~N} 4 \mathrm{O} 17 \mathrm{PS} 2, \mathrm{M}=1616.8)$.

DSPE-PEG(2000)-KK114 was prepared similarly from $3.7 \mathrm{mg}(1.3 \mu \mathrm{mol})$ of DSPE-PEG(2000)-Amine in $0.2 \mathrm{~mL}$ of dry N,N-dimethylformamide, $20 \mu \mathrm{L}(0.15$ $\mathrm{mmol})$ of triethylamine and $1.1 \mathrm{mg}(1.1 \mu \mathrm{mol})$ of KK114 NHS ester. The product was isolated with chloroform/methanol/water mixture (70/25/3) as an eluent. MS (ESI): $m / z$ (negative mode, $\%$ ) $=1820.47[\mathrm{M}-2 \mathrm{H}]^{2-}$ (for the main peak $\left.\mathrm{C}_{174} \mathrm{H}_{302} \mathrm{~F}_{4} \mathrm{~N}_{5} \mathrm{O}_{63} \mathrm{PS}_{2} \mathrm{M}=1820.48\right)$.

\section{Supported lipid bilayers (SLB)}

Glass cover slides were cleaned in a $3: 1$ mixture of concentrated $\mathrm{H}_{2} \mathrm{SO}_{4}$ and $30 \%$ $\mathrm{H}_{2} \mathrm{O}_{2}$. After acid cleaning the glass slides were extensively rinsed with pure water and then stored in water for not longer than a week. Mica substrates were prepared by cleaving thin layers of mica and gluing them to glass cover slides using optical transparent glue (Norland optical adhesive 88). The mica support was freshly prepared directly before the spin coating of the lipids. On both mica and glass supports single phase liquid disordered bilayers were made by spin coating (2000 rpm) $20 \mu 1$ of DOPC (2 $\mathrm{g} \mathrm{l}^{-1}$ in methanol-chloroform) ${ }^{38}$ After 10 min solvent evaporation under vacuum the sample was hydrated with $150 \mathrm{mM} \mathrm{NaCl}, 10 \mathrm{mM}$ TRIS/ $\mathrm{HCl} \mathrm{pH} 7.4$ and rinsed several times until a clean single bilayer remained on the surface. Ternary lipid bilayers on both supports were spin coated from a mixture of DiPhyPC, DPPC, CO in a molar ratio of $(1: 1: 0.85)$. After hydration the sample was heated to $60{ }^{\circ} \mathrm{C}$ for $5 \mathrm{~min}$ and rinsed with buffer several times until a single clean bilayer remained on the surface. As liquid disordered marker we doped the bilayer with $0.1 \mathrm{~mol} \% \mathrm{~N}$-(4,4-difluoro-5,7-dimethyl-4-bora-3a,4a-diaza-s-indacene-3-propionyl)-1,2-dihexadecanoyl-sn-glycero-3-phospho-ethanolamine (PE-BDY) (Molecular Probes, Invitrogen). For imaging concentrations in the bilayer of DSPE-KK114 or DSPE-PEG-KK114 were $0.1 \mathrm{~mol} \%$ and for STED-FCS experiments $0.01 \mathrm{~mol} \%$.

\section{Confocal and STED microscopy}

Confocal and STED imaging and fluorescence correlation spectroscopy (STEDFCS) was performed on a custom-built confocal STED microscope that was in detail described previously. ${ }^{21}$ The setup has been expanded by a fast beam scanner and a blue excitation laser $485 \mathrm{~nm}(\approx 80$ ps pulse width, LDH-P-485B, PicoQuant). Precise positioning of the laser foci in the sample and sample scanning was realized by a digital beam scanning unit (Yanus, Till-Photonics, Gräfeling, Germany) for lateral directions and a piezo scanning stage (NanoMax-TS Stage, Thorlabs, Newton, USA) for the axial direction. The fluorescence was descanned, split into two channel for detection of the red and blue fluorescence of the KK114 and Bodipy dyes, respectively, using a dichroic mirror (AHF Analysentechnik), and coupled into two multimode fiber splitters (Fiber Optic Network Technology, Surrey, Canada) with an aperture size corresponding to $1.4 \times$ the magnified excitation spot. The maximum STED power applied was $160 \mathrm{~mW}(780 \mathrm{~nm})$ measured in the back aperture of the objective (amounting to $\sim 100 \mathrm{~mW}$ at the sample) which results in a resolution or diameter of the observation area of about $40 \mathrm{~nm}$ measured either on $20 \mathrm{~nm}$ large fluorescent beads or from DSPE-KK114 diffusion in a pure DOPC bilayer. ${ }^{20,21}$

\section{STED-FCS}

Details of the STED-FCS analysis are outlined in ref. 15,21. Briefly, we recorded FCS data for different powers of the STED laser, i.e. for observation areas of different 
diameters $d$. The correlation data were fitted to: $\mathrm{G}\left(t_{c}\right)=1 / N^{*}\left(1+\left(p / h \mathrm{l}_{\mathrm{v}}\right.\right.$ Art $\left.p\right)$ ) $)$ Online $\left.* \exp \left(-t_{c} / t_{T}\right)\right) * 1 /\left(1+\left(t_{c} / \tau_{D}\right)^{\alpha}\right)$, where $N$ is the particle number $(i . e$., the mean number of fluorescent molecules in the detection volume, which is proportional to the concentration divided by the measurement volume (or area for two-dimensional samples)), $p$ is the fraction of dye that are on average in the dark triplet state, $t_{T}$ is the triplet correlation time, $\tau_{D}$ is the average transit time of the fluorescent molecules diffusing through the observation area, and $\alpha$ is the anomaly exponent, which is 1 if the diffusion is free and $<1$ for anomalous hindered diffusion. Fitting the above equation to the FCS data resulted in the dependence of the average transit time $\tau_{D}$ on the diameter $d$ of the observation area, which allowed us to determine the dependence of the apparent diffusion coefficient $D=d^{2} /\left(8 \ln (2) \tau_{D}\right)$ on $d$. The diameter $d$ of the observation area was calibrated for each power of the STED laser by STED-FCS measurements of DSPE-KK114 or DSPE-PEG-KK114 freely diffusing in a DOPC supported lipid bilayer. The molecular brightness cpp (counts-per-particle) was determined from the FCS analysis according to $c p p=N /<I>$, where $N$ is the average number of particles in the observation area (as mentioned before) and $\langle I>$ is the average count rate in $\mathrm{kHz}$ during the measurement.

\section{Partitioning coefficient}

The partitioning coefficient $\left.p_{L o}=\left\langle I_{L o}\right\rangle /\left(<I_{L o}\right\rangle+\left\langle I_{L d}\right\rangle\right)$ into the Lo phase was calculated from the average detected signal counts per image pixel $\left\langle I_{L o}\right\rangle$ and $\left\langle I_{L d}\right\rangle$ in the Lo and Ld phase, respectively. This is valid, i.e. $\left\langle I_{L o}\right\rangle$ and $\left\langle I_{L d}\right\rangle$ resemble the actual molecular distributions, since the single-molecule brightness is the same in both phases ${ }^{22}$ (compare Fig. 2C).

\section{Fluorescence lifetime}

The fluorescence lifetime was determined by fitting photon arrival time histograms that were acquired by time-correlated single-photon-counting (TCSPC). Pulsed excitation $(640 \mathrm{~nm}$ laser, $80 \mathrm{Mhz}, \approx 80$ ps pulse width, LDH-P-640B, PicoQuant, Berlin, Germany) was used to excite the fluorescent probes and the emission was detected by single-photon counting modules (avalanche photo diode SPCM-AQR-13-FC, Perkin Elmer Optoelectronics, Fremont, CA) and recorded by a TCSPC PCI card (SPC 830, Becker\&Hickl GmbH, Berlin, Germany). The fluorescence arrival time histograms were evaluated with the Matlab package Tcspcfit ${ }^{39}$ yielding the fluorescence lifetime $\tau_{F}$. The instrument response function (IRF) was determined by recording directly the laser light reflected by a mirror.

\section{Acknowledgements}

We thank Gyuzel Mitronova and Vladimir Belov (MPI-BPC Göttingen) for the synthesis of the fluorescent lipid analogues and Andreas Schönle for support with the software Imspector.

\section{References}

1 F. A. Heberle and G. W. Feigenson, Cold Spring Harbor Perspect. Biol., 2011, 3.

2 K. Simons and E. Ikonen, Nature, 1997, 387, 569-572.

3 K. Jacobson, O. G. Mouritsen and G. W. Anderson, Nat. Cell Biol., 2007, 9, 7-14.

4 D. Lingwood and K. Simons, Science, 2010, 327, 46-50.

5 S. Munro, Cell, 2003, 115, 377-388.

6 P. H. M. Lommerse, H. P. Spaink and T. Schmidt, Biochim. Biophys. Acta, Biomembr., 2004, 1664, 119-131.

7 J. F. Hancock, Nat. Rev. Mol. Cell Biol., 2006, 7, 457-462.

8 A. S. Shaw, Nat. Immunol., 2006, 7, 1139-1142.

9 M. Hao and F. R. Maxfield, J. Fluoresc., 2001, 11, 287-295. 


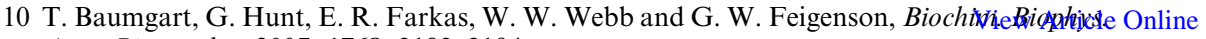
Acta, Biomembr., 2007, 1768, 2182-2194.

11 S. W. Hell, Science, 2007, 316, 1153-1158.

12 M. Bates, B. Huang and X. W. Zhuang, Curr. Opin. Chem. Biol., 2008, 12, 505-514.

13 S. W. Hell and J. Wichmann, Opt. Lett., 1994, 19, 780-782.

14 T. A. Klar, S. Jakobs, M. Dyba, A. Egner and S. W. Hell, Proc. Natl. Acad. Sci. U. S. A., 2000, 97, 8206-8210.

15 C. Eggeling, C. Ringemann, R. Medda, G. Schwarzmann, K. Sandhoff, S. Polyakova, V. N. Belov, B. Hein, C. von Middendorff, A. Schonle and S. W. Hell, Nature, 2009, 457, 1159-U1121.

16 P. F. Fahey, D. E. Koppel, L. S. Barak, D. E. Wolf, E. L. Elson and W. W. Webb, Science, 1977, 195, 305-306.

17 P. Schwille, J. Korlach and W. W. Webb, Cytometry, 1999, 36, 176-182.

18 L. Wawrezinieck, H. Rigneault, D. Marguet and P. F. Lenne, Biophys. J., 2005, 89, 40294042.

19 L. Kastrup, H. Blom, C. Eggeling and S. W. Hell, Phys. Rev. Lett., 2005, 94, 178104.

20 C. Ringemann, B. Harke, C. V. Middendorff, R. Medda, A. Honigmann, R. Wagner, M. Leutenegger, A. Schoenle, S. Hell and C. Eggeling, New J. Phys., 2009, 11, 103054.

21 V. Mueller, C. Ringemann, A. Honigmann, G. Schwarzmann, R. Medda, M. Leutenegger, S. Polyakova, V. N. Belov, S. W. Hell and C. Eggeling, Biophys. J., 2011, 101, 1651-1660.

22 E. Sezgin, I. Levental, M. Grzybek, G. Schwarzmann, V. Mueller, A. Honigmann, V. N. Belov, C. Eggeling, Ü. Coskun, K. Simons and P. Schwille, Biochim. Biophys. Acta, Biomembr., 2012, 1818, 1777-1784.

23 C. Kuo and R. M. Hochstrasser, J. Am. Chem. Soc., 2011, 133, 4664- 4667.

24 T. S. van Zanten, J. Gomez, C. Manzo, A. Cambi, J. Bucet, R. Reigad and M. F. GarciaParajo, Proc. Natl. Acad. Sci. U. S. A., 2011, 107, 15437-15442.

25 H. Mizuno, M. Abe, P. Dedecker, A. Makino, S. Rocha, Y. Ohno-Iwashita, J. Hofkens, T. Kobayashi and A. Miyawaki, Chem. Sci., 2011, 2, 1548.

26 K. Kolmakov, V. N. Belov, J. Bierwagen, C. Ringemann, V. Mueller, C. Eggeling and S. W. Hell, Chem.-Eur. J., 2010, 16, 158-166.

27 S. L. Veatch, O. Soubias, S. L. Keller and K. Gawrisch, Proc. Natl. Acad. Sci. U. S. A., 2007, 104, 17650-17655.

28 A. R. Honerkamp-Smith, P. Cicuta, M. D. Collins, S. L. Veatch, M. den Nijs, M. Schick and S. L. Keller, Biophys. J., 2008, 95, 236-246.

29 A. Honigmann, C. Walter, F. Erdmann, C. Eggeling and R. Wagner, Biophys. J., 2010, 98, 2886-2894.

30 J. Ries, S. Chiantia and P. Schwille, Biophys. J., 2009, 96, 1999-2008.

31 V. Ruprecht, S. Wieser, D. Marguet and G. J. Schuetz, Biophys. J., 2011, 100, 2839-2845.

32 M. J. Saxton, Biophys. J., 1994, 66, 394-401.

33 T. Baumgart, A. T. Hammond, P. Sengupta, S. T. Hess, D. A. Holowka, B. A. Baird and W. W. Webb, Proc. Natl. Acad. Sci. U. S. A., 2007, 104, 3165-3170.

34 D. Lingwood, J. Ries, P. Schwille and K. Simons, Proc. Natl. Acad. Sci. U. S. A., 2008, 105, 10005-10010.

35 W. Ngamsaad, S. May, A. J. Wagner and W. Triampo, Soft Matter, 2011, 7, 2848.

36 S. J. Sahl, M. Leutenegger, M. Hilbert, S. W. Hell and C. Eggeling, Proc. Natl. Acad. Sci. U. S. A., 2010, 107, 6829-6834.

37 S. L. Veatch and S. L. Keller, Biochim. Biophys. Acta, Mol. Cell Res., 2005, 1746, 172-185.

38 M. H. Jensen, E. J. Morris and A. C. Simonsen, Langmuir, 2007, 23, 8135-8141.

39 J. Enderlein and R. Erdmann, Opt. Commun., 1997, 134, 371-378. 